\title{
Arnold-Chiari malformation and nystagmus of skew
}

\author{
Christina Pieh, Irene Gottlob
}

\begin{abstract}
The Arnold-Chiari malfomation is typically associated with downbeat nystagmus. Eye movement recordings in two patients with Arnold-Chiari malfomation type 1 showed, in addition to downbeat and gaze evoked nystagmus, intermittent nystagmus of skew. To date this finding has not been reported in association with Arnold-Chiari malfomation. Nystagmus of skew should raise the suspicion of Arnold-Chiari malfomation and prompt sagittal head MRI examination.

(F Neurol Neurosurg Psychiatry 2000;69:124-126)
\end{abstract}

Keywords: Arnold-Chiari malformation; nystagmus; nystagmus of skew; eye movement recordings

In the Arnold-Chiari malformation a part of the brainstem and the cerebellum are herniated into the cervical vertebral canal. The malformation is classified according to the anatomical changes seen. Type I consists of downward displacement of the cerebellar tonsils only. Type II involves displacement of the cerebellum, lower pons, and medulla, and is usually diagnosed in childhood. Type III involves a cerebellar herniation into a high cervical meningocele. Clinical findings include oscillopsia, impaired smooth pursuit, and OKN, and in many cases downbeat nystagmus ${ }^{12}$ which can be the salient clinical manifestation. ${ }^{3}$ Eye movement recordings in patients with Arnold-Chiari malformation are useful for characterisation of downbeat $^{5}$ and less common forms of nystagmus. ${ }^{6}$

We report on two patients with ArnoldChiari malformation who presented with vertical jerk nystagmus. Eye movement recordings disclosed nystagmus with one eye beating upwards whereas the other eye was simultaneously beating downwards, which we term "nystagmus of skew". To our knowledge, this has not been described in the literature.

Correspondence to: Professor Irene Gottlob, Leicester Royal Infirmary, Department of

Ophthalmology, LE1 5WW

Leicester, UK

ig15@le.ac.uk

Received 20October 1999 and in revised form 28 February 2000

Accepted 6 March 2000
Clinical evaluation of the two patients included complete ophthalmological and neurological examination. Eye movements in the horizontal and vertical planes were recorded by Robinson's electromagnetic scleral search coil technique. ${ }^{7}$ Data were converted from analog to digital and recorded on a computer with a sampling rate of $230 \mathrm{~Hz}$.

Brain MRI was performed with a $1.5 \mathrm{~T}$ scanner. Sagittal views of T1 weighted images were obtained.

Case 1

A 46 year old woman with no history of ophthalmological disease experienced decreased vision after recovery from hip replacement surgery. The operation was performed under general anaesthesia, after an attempted epidural anaesthesia had failed, probably due to penetration into the subarachnoid space. The patient reported blurred vision and oscillopsia on downgaze, but did not complain of double vision. Ophthalmological examination showed decreased near visual acuity of 0.6 in each eye. Distance visual acuity was 1.0 in each eye. Examination of eye motility showed slight bilateral weakness of the inferior and lateral rectus muscles. Horizontal or vertical optokinetic nystagmus could not be elicited. The vestibular ocular reflex was normal. Smooth pursuit was saccadic in all directions. Downbeat nystagmus on down and lateral gaze was seen clinically. A torsional component was not detected with the slit lamp or the ophthalmoscope. However, the nystagmus amplitude was small and, therefore, we cannot exclude a torsional component. The remainder of the neurological examination was normal. Brain MRI showed a $2 \mathrm{~cm}$ herniation of the cerebellar tonsils through the foramen magnum. Nystagmus diminished after several months, and only small nystagmus persisted on downgaze. No surgery was performed.

When first referred to our clinic, eye movement recordings showed periods of intermittent, associated downbeat nystagmus of both eyes in primary fixation which increased on lateral and downgaze (figure A). On upgaze, upbeat nystagmus of low frequency and amplitude could be seen. On lateral gaze, additional horizontal, associated, low frequency gaze evoked nystagmus was noted (figure B). During the same recording session periods of vertical nystagmus of skew were detected. Downbeat nystagmus of the right eye and upbeat nystagmus of the left eye were seen at the same time during primary fixation. Both eyes showed oscillations of about the same amplitude and frequency. Slow phases of 

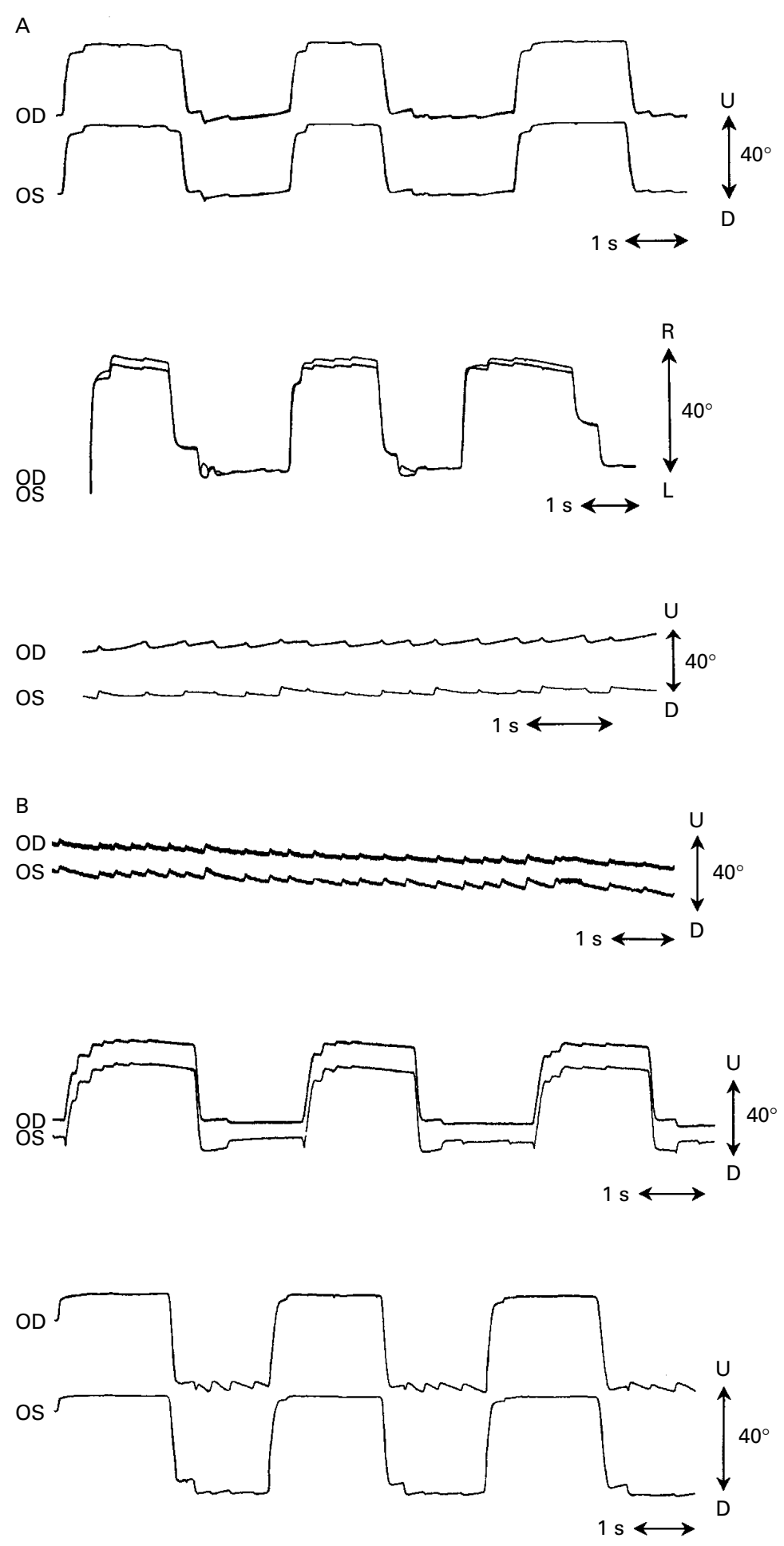

(A) Original search coil recordings of patient 1. Top: vertical position of the right (top trace) and left (bottom trace) eye during $40^{\circ}$ vertical saccades $\left(20^{\circ}\right.$ up and down from primary position). Associated downbeat nystagmus is seen on downgaze (2-4 $\left.4^{\circ}, 2-3 \mathrm{~Hz}\right)$. Centre: horizontal position of the right (top trace) and left (bottom trace) eye during $40^{\circ}$ horizontal saccades (20 right and left from primary position). Associated nystagmus to the right of $2-4^{\circ}$ and 3-4 Hz is seen on lateral right gaze. Bottom: vertical position of the right (top trace) and left (bottom trace) eye during downgaze and left gaze. Downbeat nystagmus of the right eye and upbeat nystagmus of the left eye with low amplitude (2-4) and frequency $(2-3 \mathrm{~Hz})$ is seen. (B) Original search coil recordings of patient 2. Top: Vertical position of the right (top trace) and left (bottom trace) eye during $20^{\circ}$ upgaze. Associated upbeat nystagmus of low amplitude (2-4 ) and low frequency (2-3 Hz) of the right and left eye is seen. Centre: vertical position of the right (top trace) and left (bottom trace) eye during $40^{\circ}$ vertical saccades ( $20^{\circ}$ up and down from primary position).

Associated upbeat nystagmus of $2-3^{\circ}$ and $4 \mathrm{~Hz}$ is seen on upgaze. Bottom: vertical position of the right (top trace) and left (bottom trace) eye during $40^{\circ}$ vertical saccades $\left(20^{\circ}\right.$ up and down from primary position). Upbeat nystagmus of the right eye and downbeat nystagmus of the left eye with low amplitude $\left(5^{\circ}\right)$ and low frequency $(4 \mathrm{~Hz})$ is seen on downgaze. nystagmus were of constant velocity (figure A, bottom).

Case 2

A 36 year old woman presented with a brief history of progressive decrease of visual acuity, difficulty in walking, and vertigo induced by turning the head in any direction. She complained of blurred vision, but did not report oscillopsia. Visual acuity was 0.6 in each eye for near and distance with correction. This was most likely due to an ametropic amblyopia caused by astigmatism, for which she had never been corrected. On clinical examination smooth pursuit was saccadic in all directions. Optokinetic nystagmus seemed normal. Downbeat and right beating nystagmus were noted in primary position and on downgaze. On lateral gaze, an additional rotatory component was found. Neurological examination was otherwise normal. Brain MRI showed an ArnoldChiari malformation type I with cerebellar tonsils $1.5 \mathrm{~cm}$ below the level of the foramen magnum. No associated structural lesions or basilar impressions were noted. A posterior fossa decompression, an upper cervical laminectomy, and an expansive duraplasty were performed, which led to gradual relief of the symptoms. The nystagmus diminished within a period of 3 months.

Eye movement recordings before surgery showed associated jerk nystagmus to the right in the primary position. Downgaze triggered downbeat nystagmus which was associated part of the time. On lateral gaze, jerk nystagmus in the direction of the gaze and downbeat nystagmus were found. On upgaze she had a low amplitude upbeating and right beating nystagmus (figure B, top and centre). During downgaze and right lateral gaze, however, phases of nystagmus of skew with the right eye beating up and the left eye simultaneously beating down were seen (figure B, bottom).

\section{Discussion}

In patient 1 the probable penetration of the subarachnoid space during anaesthesia may have triggered the onset of clinical symptoms, consistent with previously reported development of nystagmus and oscillopsia in ArnoldChiari malformation after lumbar puncture ${ }^{8}$ which may accentuate a craniospinal pressure gradient and promote compression, displacement, or distortion of vestibulocerebellar structures.

In addition to downbeat nystagmus, which is usually associated with structural lesions at the cervicomedullary junction that may affect the cerebellum, lower brain stem, and spinal cord, several other eye movement abnormalities such as periodic alternating nystagmus and horizontal, torsional, or gaze evoked nystagmus, have been described in Arnold-Chiari malformation. In a report of the disease with convergence nystagmus the authors postulated that it was due to a combination of mechanical distortion of the brainstem and cerebellum and abnormal transmission of CSF pressure to the aqueductal region. ${ }^{6}$ See-saw nystagmus has been described in association with Arnold- 
Chiari malformation in one case, where nystagmus was present in the primary position and in all directions of gaze, but was accentuated during left lateral gaze and was larger in the left eye. ${ }^{9}$ As one of our patients showed a rotatory component of nystagmus during clinical examination, a fine see-saw nystagmus, which is not recordable with the two dimensional coil, cannot be excluded. In this case the interstitial nucleus of Cajal would most likely be involved. Primary dissociated vertical jerk nystagmus is rare and has been described in association with internuclear ophthalmoplegia, suggesting a lesion of the midbrain in the region of the medial longitudinal fasciculus. ${ }^{10} \mathrm{~A}$ similar abnormality may also have contributed to the eye movement disorder in our patients.

The anatomical substrate of a pure skew nystagmus is not known. Because cerebellar dysfunction may be associated with skew deviation and downbeat nystagmus and is also a feature of Arnold-Chiari malformation, it is possible that the malformation impinges on the oculomotor muscle connectivity at cerebellar levels.

In conclusion, we report the unusual association of nystagmus of skew with Arnold-Chiari malformation. It is possible that nystagmus of skew is more common in Arnold-Chiari malformation than previously thought, and eye movement recordings may detect such nystagmus. Arnold-Chiari malformation should be considered in patients in whom clinical evaluation or eye movement recordings demonstrate nystagmus of skew and should prompt a sagittal head MRI examination.

Supported by the Swiss National Science Foundation (NR. $32-52503.97$ ) and by the OPOS Foundation.

1 Saez RT, Onofrio BM, Yanagihara T. Experience with Arnold-Chiari malformation, 1960-70 ff Neurosurg $1976 ; 45: 416-22$

2 Chait GE, Barber HO. Arnold-Chiari malformation-some otoneurological features. F Otolaryngol 1979;8:65-70.

3 Corkill G, Vijayan N. Saccadic visual suppression and down-beat nystagmus: a case of Arnold-Chiari malformation. Surg Neurol 1976;5:95-6.

4 Faria MA, Spector RH, Tindall GT. Downbeat nystagmus as the salient manifestation of the Arnold-Chiari malformation. Surg Neurol 1980;13:333-6.

5 Zee DS, Friendlich AR, Robinson DA. The mechanism of downbeat nystagmus. Arch Neurol 1974;30:227-37.

6 Mossman SS, Bronstein AM, Gresty MA, et al. ConverMossman SS, Bronstein AM, Gresty MA, et al. Conver-
gence nystagmus associated with Arnold-Chiari malformagence nystagmus associated with
tion. Arch Neurol 1990;47:357-9.

7 Robinson DA, Gordon JL, Gordon SE. A model of the smooth pursuit eye movement system. Biol Cybern 1986;55:43-57.

8 Barton JJS, Sharpe JA. Oscillopsia and horizontal nystagmus with accelerating slow phases following lumbar punc33:418-21.

9 Zimmerman CF, Roach ES, Troost BT. See-saw nystagmus associated with Chiari malformation. Arch Neurol 1986;43: 299-300.

10 Marshall RS, Sacco RL, Kreuger R, et al. Dissociated vertical nystagmus and internuclear ophthalmolplegia from a midbrain infarction. Arch Neurol 1991;48:1304-5. 